\title{
Association of Neck Disability and Upper Extremity Musculoskeletal Discomfort in Text Neck Syndrome among Adults
}

\section{Bhavna Anand ${ }^{1 *}$, Sara Nishat ${ }^{1}$, Natasha Khanna ${ }^{2}$, Shubhi Kulshrestha ${ }^{1}$ and Sukriti Raj ${ }^{1}$}

${ }^{1}$ Amity Institute of Physiotherapy, Amity University Noida, UP, India

${ }^{2}$ Aarogya Physiotherapy and Rehabilitation, Sarita Vihar, New Delhi, India

*Corresponding Author: Bhavna Anand, Amity Institute of Physiotherapy, Amity University Noida, UP, India.
Received: September 01, 2021

Published: September 23, 2021

(C) All rights are reserved by Bhavna Anand., et al.

\begin{abstract}
Background: Text neck syndrome is a terminology used to describe a repetitive stress injury which occurs when individuals keep their head and neck flexed downwards. This syndrome is known to occur during activities like texting, typing when the neck is bent for extended periods and leads to excessive amount of pain. The aim is to check the association of neck disability and upper extremity musculoskeletal discomfort in text neck syndrome among adults age group 18 - 45 years using smartphones and other electronic devices more than 5 hours a day for more than a year.

Methods: 113 participants took part in survey out of which 22 were excluded as they didn't fulfil the criteria of inclusion. Data was collected from 91 participants and two standard questionnaires - Neck Disability Index and Upper Extremity Functional Index- 15 were used for data collection. Data was interpreted using Microsoft excel and correlation was found using Pearson coefficient.

Results: There was slightly positive association between neck disability and upper extremity discomfort and out of 91 participants, 80 had some characteristic of neck disability. The present study shows prevalence of neck disability due to text neck syndrome to be around $28.17 \%$ in the 18 - 45 age group by using NDI scale. Most affected age group was 21 years.
\end{abstract}

Conclusion: The study shows weak positive association between neck disability and upper extremity musculoskeletal discomfort in text neck among adults with $\mathrm{r}$ value (0.343).

Keywords: Association; Text Neck Syndrome; Upper Extremity Discomfort; Neck Pain; Neck Disability

\section{Introduction}

Text neck syndrome is a terminology outlining a repetitive stress injury which occurs when individuals keep their head and neck flexed downwards [1]. It is known to occur during activities like texting, typing when the neck is bent for extended periods and leads to excessive amount of pain in shoulder and stiffness, spam in back muscles [1]. It can be often appertained to tension in the cervical spine musculature which can lead to persistent and longlasting pain especially in young people as an outcome of greatest exposure to handheld devices [2]. As claimed by research, there is connection and coexistence of innumerable co morbidities existing with text neck leading to chronic pain conditions if not treated on 
time [3]. There is a corresponding increase in the angle of flexion and the load acting on the spine. From 10 - $12 \mathrm{lbs}$ force acting at about zero degree of neck tilt, to as much as 60 pounds load acting at 60 degrees of neck flexion [1].

Repetitive movements, high amounts of physical demands on the human body, postures that have been maintained for extended hours and defective ergonomic design are often correlated with complaints of pain and discomfort which not only occur in neck but in regions of upper limb like shoulder, arm, thumb and fingers [4]. A study supported the interrelatedness of typing style and type of gadget to the activity of upper limb musculature which also guides the posture of cervical region [5]. There is also a relation between the duration as well as the regularity with which the handheld devices are used leading to pain in musculature of upper extremity [6]. The angle at which the head is bent or flexed is dependent on variety of tasks like texting, watching video or web browsing [7]. Most amount of flexion occurs in texting hence leading to pain and fatigue and this posture during texting is usually adopted in order to limit the biomechanical loading on muscles of shoulder which would occur if the placement of mobile phone was done at a higher level [7].

There is a possibility of association of neck disability to sustained posture of neck in downward position which can lead to load and stress being obtruded on spine and other pathologies can also initiate [8].

There are numerous symptoms which are associated with text neck such as pain of neck and tightness caused by sustained position which leads to problems in the musculature, shoulder spams and tightness which is very commonly seen creating excessive load on the muscles of arm, hence performance will be affected as productivity decreases and fatigue increases due to lactic acid formation. Long term existence of forward head posture and round shoulders leads to spinal degeneration, compression and various other pathologies of spine and upper extremity $[2,9,10]$. Management protocol includes a combination of ergonomic, physical therapeutic and lifestyle moderation instructions. Ergonomic guidelines have to be followed as a preventive strategy hence it is essential to have support under forearm while typing, extreme flexion of neck has to be avoided and one should avoid typing at high velocity [10]. Students with addiction to mobile phone devices exhibit progres- sion of muscular discomfort in neck region and hand especially the thumb which may lead to long term disability [11]. Cervical spine is exposed to notable musculoskeletal risk factors due to extended periods of devices usage and the dynamics of cervical vertebrae and cervical extensor muscles tends to be distinguishable in various conditions while using typing devices which necessitates the consideration of human posture friendly design while development of such devices [12]. New researches have established that neck pain and related discomfort has been increasing because of ongoing alteration in pattern of working and lifestyle caused by Covid-19 pandemic and best method to intercept such pain and syndrome is by awareness among population about the basic posture correction and regular exercises [13].

\section{Aim and Objective}

This study is to check the association between neck disability and upper extremity musculoskeletal discomforts in text neck syndrome among people of age group 18 - 45 years using smartphones, tablets and laptops more than 5 hours daily for more than a year.

\section{Research Design and Methodology}

Research design: Quantitative research design is used with a close end questionnaire- Survey based study. Questionnaire was given in google forms and then data collection, tabulation analysis and interpretation was done by Microsoft excel. 91 participants in age group of 18 - 45 years. Non-probability sampling was done. MS excel Pearson correlation coefficient was used to find out the association.

\section{Selection criteria}

Inclusion criteria: Subjects of age group 18 - 45 years. Participants using smartphones and tech devices for more than 5 hours daily beyond a year (Gracias LA., et al. 2019). Subjects who agreed to fill the consent form (Gracias LA., et al. 2019). One who can read or comprehend English.

Exclusion criteria: Those who are not able to read or comprehend English. Subjects who didn't use smartphone and other electronic devices for more than 5 hours daily beyond 12 months. Subjects who didn't agreed to fill the consent form (Gracias LA., et al. 2019). Subjects with previous history of neck trauma, upper limb pathology, surgery, systemic illness, neuropathic condition, fracture, 
vertigo or neurological conditions affecting upper and lower limb were excluded. Subjects taking part in recreational activities which includes repeated movements of upper limb or neck for past 3 months (Kalirathinam D., et al. 2019).

\section{Procedure}

Participants were explained about the intention and motive of study and those eligible fulfilling the criteria of inclusion were given the consent form. Demographic details and questions about occurrence of pain, number of hours spent on phone or electronic devices were asked. Form further consisted of 2 questionnaires neck disability index questionnaire (NDI) and upper extremity functional index - 15 (UEFI) to collect information regarding neck disability and upper extremity discomfort. Pearson's correlation coefficient was accustomed to scrutinize the linkage between the two.

\section{Results}

The survey form was filled by 113 participants and 22 were excluded as they didn't fulfil the inclusion criteria. 91 feedbacks were assembled in total. Neck disability index was used to analyse neck disability among adults due to text neck syndrome and it revealed there was $28.17 \%$ prevalence of the same.

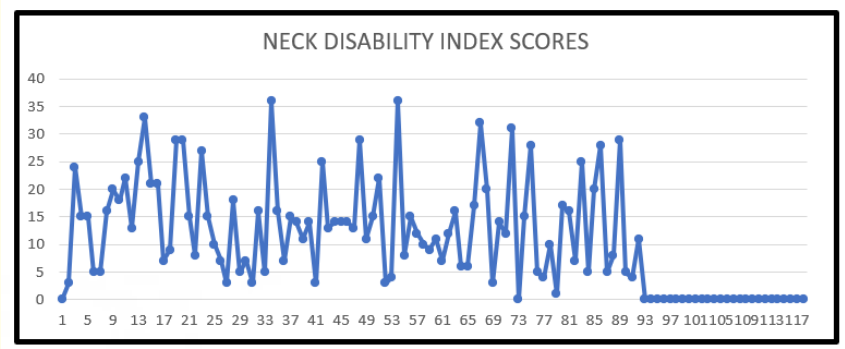

Figure 1

Majority of participants, 40 out of 91 are in the category of mild disability (43.95\%) followed by 25 in moderate disability (27.47\%), 13 in severe category (14.28\%), 11 in no disability category $(12.08 \%)$ and lastly 2 under complete disability (2.19\%).

In this 10-part questionnaire, the most severely afflicted component was sleeping followed by headaches, driving, concentra-

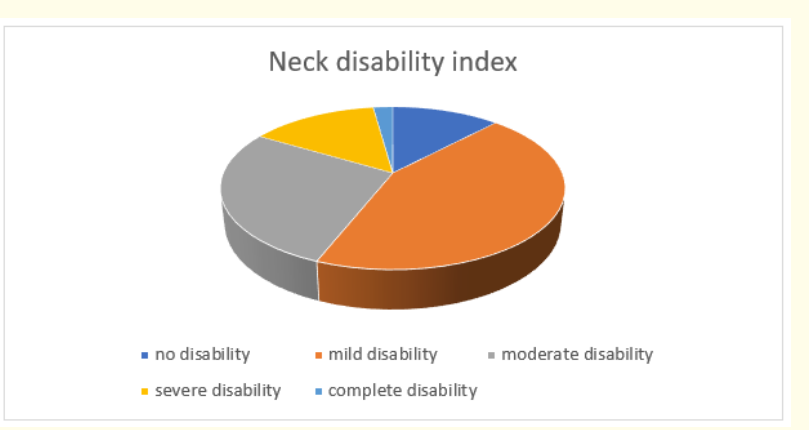

Figure 2

tion, work and pain intensity equally, lifting and reading equally and lastly personal care and recreation components weren't severely affected at all. Upper extremity discomfort was measured using upper extremity functional index-15 and the overall percentage affected was $24.35 \%$. In the $0-100$ interval level scale, only 8 participants have a score below 50 wherein 100 is perceived as best state and 0 as the worst state.

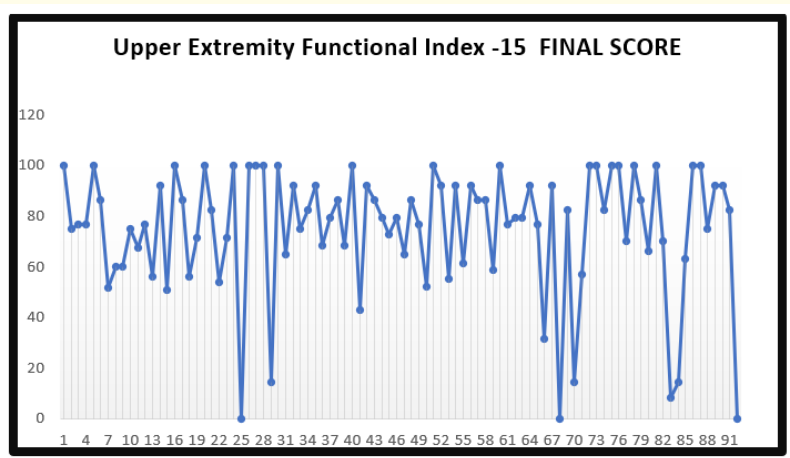

Figure 3

Correlation was done using Pearson's coefficient to see the association between neck disability and upper extremity musculoskeletal discomfort in text neck among adults. The ' $r$ ' value was found to 0.343 thus indicating weak to moderately positive linkage of the two components. 
Female to male ratio of the members who participated was 69:22. Mean age of the subjects is 21 years. Majority of subjects who took part were 21 years of age $(27.5 \%)$ followed by 20 years (25.3\%). 90.1\% are students. All the participants used electronic gadgets beyond 5 hours every day apart from calling but some used for more than 10 and 17 hours. Those who used for less than five hours were excluded. From a total of 91subjects, 51 held their device at chest level, 30 at abdominal while only 10 held at their eye level. Out of 91 participants, neck pain was felt occasionally $(37.4 \%)$ followed by rarely $(24.2 \%)$ and often $(22 \%)$ due to prolonged use of electronic devices. Upper extremity discomfort and pain had a similar pattern occasionally (35.2\%) followed by rarely (34.1\%) and often (19.8\%). Neck stiffness was experienced occasionally $38.5 \%$, often $23.1 \%$ followed by rarely $22 \%$. The most common site of pain in upper limb was upper back region experienced by $41.9 \%$ participants followed by shoulders $34.9 \%$ and subsequently hand and arm. It was also seen that maximum pain was felt in right extremity (57.6\%) which was the dominant hand for $91.2 \%$ participants.

\section{Discussion}

The foremost motive was to understand the influence of text neck syndrome due to long term exposure of electronic devices and hours spent on texting, typing, watching videos etc which require bending of neck to a certain angle and thus emphasising stress in the cervical region and additionally causing headaches, soreness in muscles, pain and even spinal degeneration if the sustained posture isn't corrected within time. All the participants used phones and devices for more than five hours a day apart from calling. The main intention was to find and check if there was a link or any connection between neck disability and upper extremity discomfort in text neck among adults who are using smartphones and other devices for more than 5 hours every day. The study demonstrates weakly moderate alliance linking these two elements in text neck syndrome among adults (0.343). The numbers spent on phones and other devices have remarkably seen an upsurge due to the covid-19 pandemic as we have become more dependent on online and virtual platforms. Out of 91 participants who took part and fulfilled the inclusion criteria, 80 fell in some category of neck disability and only 11 didn't have any disability and the presence was estimated to be $28.17 \%$. The most persistent regions of soreness and pain have been upper back area and shoulder and it was also disclosed that maximum of them had symptoms of discomfort in the dominant hand which was right.

A corresponding study was done by Sathya P. in June 2020 which concluded that the extensiveness of text neck syndrome was $32 \%$ amid a group of young adults in which females were more afflicted about $80 \%$ and most of them were 22 years old. The purpose was to see the presence of text neck syndrome among young adults in which hundred university students were taken as subjects and a demographic data form along with Neck Disability Index questionnaire was filled by them with prior consent. The most affected constituent in the questionnaire was headache succeeded by sleeping [14]. A study done by Kalirathinam D in April 2017 showed positive interlinkage of pain and discomfort in upper arm, forearm and hand to neck region with the use of mobile phone by carrying out a survey with a self-administered questionnaire. The aggregate consequence shows that among 244 students who took part in the study, 49 showed symptoms of upper limb and neck region discomfort. The order of proportion from maximum to minimum was neck symptoms followed by right shoulder and then left shoulder symptoms [15].

The data derived from a study done by Damasceno GM in 2018 shows contrasting results from our study with no alliance found between text neck and pain in neck by regression analysis in 18 21 age group and even assessed the neck posture [16]. Also, it is absolutely crucial to include breaks and realise that correct posture during activities of texting, working on electronic devices along with implying the ergonomic guidelines can help prevent the pain caused by text neck syndrome and also prevent long term consequences.

Limitations of this study includes small sample size, online collection of data due to ongoing Covid -19 pandemic.

\section{Conclusion}

This current study exhibits prevalence of neck disability because of text neck syndrome to be around $28.17 \%$ in the 18 - 45 age group by using NDI scale. Upper extremity discomfort was found to be $24.35 \%$ through upper extremity functional index -15 scores and the most impacted age group was 20 - 21 years. There was a weak positive interrelation of neck disability and upper extremity discomfort in text neck among adults with r value (0.343). 


\section{Conflict of Interest}

Authors have no conflict of interest to report.

\section{Bibliography}

1. Neupane S., et al. "Text Neck Syndrome - Systematic Review". Imperial Journal of Interdisciplinary Research (2017): 141-148.

2. Nizar Abdul Majeed Kutty. "Text neck: a global epidemic of the modern era". MOJ Yoga and Physical Therapy (2019): 14-16.

3. M Vijayakumar., et al. "Assessment of Co- Morbid Factors associated with Text neck syndrome among Mobile Phone users". International Journal of Scientific Research in Science and Technology (2018): 38-46.

4. Berolo S., et al. "Musculoskeletal symptoms among mobile hand-held device users and their relationship to device use: A preliminary study in a Canadian university population". Applied Ergonomics (2011): 371-378.

5. Kietrys DM., et al. "Mobile input device type, texting style and screen size influence upper extremity and trapezius muscle activity, and cervical posture while texting". Applied Ergonomics (2015): 98-104.

6. Balakrishnan R., et al. "An extensive usage of hand held devices will lead to musculoskeletal disorder of upper extremity among student in AMU: A survey method". International Journal of Physical Education, Sports and Health 3 (2016): 368-372.

7. Lee S., et al. "Head flexion angle while using a smartphone". Ergonomics (2015): 220-226.

8. AlAbdulwahab Sami S., et al. "Smartphone use addiction can cause neck disability". Musculoskeletal Care 15.1 (2017): 1012.

9. David DeWitt. “Text Neck Syndrome and Diagnosis” (2018).

10. Gustafsson E. "Ergonomic recommendations when texting on mobile phones". Work 41.1 (2012): 5705-5706.

11. Shah PP., et al. "Correlation of smartphone use addiction with text neck syndrome and SMS thumb in physiotherapy students". International Journal of Community Medicine and Public Health (2018).
12. Ning X., et al. "Neck kinematics and muscle activity during mobile device operations". International Journal of Industrial Ergonomics (2015): 10-15.

13. Bhattacharya S. "Texting neck" or "iNeck pain" syndrome - An emerging public health threat: In the era of NEW NORMAL". Journal of Primary Care Specialists (2021): 1-3.

14. Sathya P., et al. "Prevalence of Text Neck Syndrome in youngadult population". Ijmaes 6.2 (2020): 749-759.

15. Association between the usage of Smartphone as the risk factor for the prevalence of upper extremity and neck symptoms among University students: A cross - sectional survey based study". Research Journal of Pharmacy and Technology (2017): 1184-1190.

16. Damasceno GM., et al. "Text neck and neck pain in 18-21-yearold young adults". European Spine Journal: Official Publication of the European Spine Society, the European Spinal Deformity Society, and the European Section of the Cervical Spine Research Society (2018): 1249-1254.

\section{Volume 4 Issue 10 October 2021 (C) All rights are reserved by Bhavna Anand., et al.}

